\title{
Antibiotic Resistance and Molecular Epidemiology of Vancomycin-Resistant Enterococci in a Tertiary Care Hospital in Turkey
}

This article was published in the following Dove Press journal:

Infection and Drug Resistance

\author{
Nergis Asgin (1D' \\ Baris Otlu ${ }^{2}$ \\ 'Department of Medical Microbiology, \\ Faculty of Medicine, Karabuk University, \\ Karabuk, Turkey; ${ }^{2}$ Department of Medical \\ Microbiology, Faculty of Medicine, Inonu \\ University, Malatya, Turkey
}

Purpose: Vancomycin-resistant enterococci (VRE) have become a global health threat in the last two decades. In this study, we aimed to determine antibiotic resistance using phenotypic and genotypic methods in VRE strains obtained from inpatients and to investigate clonal relatedness among strains.

Methods: Identification and antibiotic susceptibility of 47 VRE strains obtained from inpatients at Karabuk University Hospital from 2014 to 2015 were determined using the BD Phoenix ${ }^{\mathrm{TM}}$ automated microbiology system. Vancomycin resistance genes (Van A and B) were detected by polymerase chain reaction. Clonal relatedness among the strains was evaluated by pulsed-field gel electrophoresis (PFGE).

Results: All 47 VRE strains obtained from rectal $(n=35)$, blood $(n=7)$, and urine $(n=5)$ samples were confirmed as Enterococcus faecium; they were resistant to ampicillin, gentamicin, vancomycin, and teicoplanin. One E. faecium isolate was intermediately resistant to linezolid. No strain was resistant to quinupristin-dalfopristin or daptomycin. Only vanA was detected among strains. According to the PFGE results, 31 of 47 strains were clonally related with a clustering rate of $66 \%$. No common clone was detected.

Conclusion: VRE infections are associated with high mortality, morbidity, and healthcare expenditures. Increasing resistance to last-line drugs, such as linezolid and daptomycin, among VRE strains is a great concern. Therefore, comprehensive measures should be performed to reduce VRE colonization. Although there was no common clone VRE outbreak, polyclonal spread was observed in our hospital. The high clustering rate indicated cross-contamination. Thus, a more effective infection control program should be implemented.

Keywords: antimicrobial resistance, Enterococcus faecium, gastrointestinal tract, linezolid resistance, pulsed-field gel electrophoresis

\section{Introduction}

Enterococci are part of the normal intestinal flora of humans and are a capable of dominating the gut microbiota of long-term hospitalized patients who receive broad-spectrum antibiotics. ${ }^{1,2}$

Following gastrointestinal colonization, enterococci can lead to bloodstream Karabuk University, Faculty of Medicine, Department of Medical Microbiology,

Baliklarkayasi Mevkii Demir Celik Kampusu, Karabuk 78050, Turkey

Tel +903704189446

$\mathrm{Fax}+903704189299$

Email drnasgin@gmail.com infections, infective endocarditis, and intra-abdominal/pelvic abscess in critically ill patients. ${ }^{1,3-5}$ Enterococci can grow under a wide range of temperatures $\left(10^{\circ} \mathrm{C}-45^{\circ} \mathrm{C}\right)$ and are resistant to dry conditions; therefore, the bacteria can persist for long periods in hospital environments and spread easily among patients. ${ }^{2,3,6}$ 
Enterococci are intrinsically resistant to cephalosporins and low levels of aminoglycosides. In addition, because of increasing resistance to vancomycin as well as high-level aminoglycoside resistance, the treatment options for vancomycin-resistant enterococci (VRE) are limited. Enterococci acquire resistance to glycopeptides via eight vancomycinrelated genetic elements: vanA, vanB, vanD, vanE, vanG, vanL, vanM, and vanN. ${ }^{5-7}$ The vanA gene is located within the Tn1546 transposon and confers transferable acquired resistance. Moreover, these genetic elements impart highgrade resistance to vancomycin and teicoplanin. The vanB phenotype confers different levels of vancomycin resistance and teicoplanin susceptibility. Nosocomial VRE infections can develop either endogenously, where colonization in critically ill patients is followed by invasive infection, or exogenously, in which the bacteria are transmitted via healthcare workers or contact with contaminated instruments and inanimate surfaces. ${ }^{1,3-6}$ To prevent VREassociated nosocomial infections, it is crucial to determine the source of infection and routes of transmission as well as identification of patients colonized with VRE.

However, there have been relatively few reports on molecular characterization and clonal epidemiology of VRE in Turkey. Therefore, we aimed to characterize the phenotypic and genotypic resistance profiles of 47 VRE strains obtained from hospitalized patients in Turkey and to evaluate the clonal relatedness among strains by PFGE.

\section{Materials and Methods Study Design}

This cross-sectional study was conducted between October 2016 and March 2017 at the Karabuk University Training and Research Hospital, a 440-bed tertiary hospital in the city of Karabuk, Turkey, which is located in the western Black Sea region.

\section{Bacterial Strains}

A total of 47 VRE strains were included in this study. The VRE strains were part of the routine hospital laboratory procedure and obtained from the clinical and rectal swab (RS) samples of inpatients between May 2014 and October 2015. The strains were stored in tryptic soy broth supplemented with $10 \%$ glycerol at $-80^{\circ} \mathrm{C}$ until use. Only one strain from each patient was included. Repetitive, non-VRE, contaminated, or unanimated strains were excluded. Molecular analysis of the VRE strains was performed at the Molecular Microbiology Laboratory of Inonu University (Malatya, Turkey).

\section{Identification and Antibiotic Susceptibility Testing}

In a microbiology laboratory setting, the RS samples were inoculated on Enterococcosel agar (Becton Dickinson and Company, Franklin Lakes, NJ, USA) and clinical specimens were cultured on Columbia agar supplemented with $5 \%$ sheep blood, eosin-methylene blue agar, and chocolate agar (all, Becton Dickinson and Company). After incubation for $24-48 \mathrm{~h}$ at $35^{\circ} \mathrm{C}$, black-colored colonies that grew on Enterococcosel agar were subcultured on Columbia agar supplemented with 5\% sheep blood. Colonies cultured on blood agar were assessed by Gram staining and pyrrolidinyl aminopeptidase (PYR) and catalase tests. The identification and antibiotic susceptibility of catalase-negative, PYR-positive, and Gram-positive cocci were determined using the BD Phoenix ${ }^{\mathrm{TM}}$ Automated Identification and Susceptibility Testing System (Becton Dickinson and Company BD, Sparks, MD, USA). The identification of strains was also confirmed by the matrix-assisted laser desorption/ionization time-of-flight (MALDI-TOF) method with the VITEK ${ }^{\circledR}$-MS device (BioMérieux, Marcy-l'Étoile, France). Vancomycin resistance was also confirmed using gradient minimum inhibitory concentration method with E-test strips (BioMérieux, Marcy-l'Étoile, France). The antibiotic susceptibility of strains was determined according to the Clinical and Laboratory Standards Institute guidelines. ${ }^{9}$ Enterococcus faecalis ATCC 29212 was used for quality control.

\section{Determination of Vancomycin-Resistant Genes}

Genomic DNA was isolated using the QIAsymphony DSP Virus/Pathogen Kit and the QIAsymphony SP Automated Nucleic Acid Purification System in accordance with the manufacturer's instructions (Qiagen, Inc., Valencia, CA, USA). The DNA samples were stored at$80^{\circ} \mathrm{C}$ until use. The van $\mathrm{A}$ and vanB resistance genes were evaluated using an in-house polymerase chain reaction (PCR) method, as previously described, ${ }^{10}$ and a GeneAmp $^{\circledR}$ PCR System 9700 thermal cycler (Applied Biosystems, Carlsbad, CA, USA). The amplification conditions were as follows: initial denaturation for $10 \mathrm{~min}$ at $94^{\circ} \mathrm{C}$, followed by 40 cycles of denaturation at $94^{\circ} \mathrm{C}$ for $1 \mathrm{~min}$, annealing at $57^{\circ} \mathrm{C}$ for $1 \mathrm{~min}$, extension at $72^{\circ} \mathrm{C}$ for $1.5 \mathrm{~min}$, and a final extension at $72^{\circ} \mathrm{C}$ for $10 \mathrm{~min}$. 
The amplified products were separated by electrophoresis for $1 \mathrm{~h}$ at $100 \mathrm{~V}$ in a $1.5 \%$ agarose gel, which was then stained with ethidium bromide and imaged using the Kodak Gel Logic 200 Imaging System (Eastman Kodak Company, Rochester, NY, USA) under ultraviolet light. The band size of each gene was compared with a 100 bp DNA ladder (New England Biolabs, Ipswich, MA, USA).

\section{Detection of Clonal Relationship Among Isolates}

To determine the clonal relationship among isolates, PFGE typing was conducted using a previously described method $^{11}$ with some modifications. In brief, the samples were digested with the SmaI restriction enzyme (New England Biolabs) and separated by electrophoresis using the CHEF-DR ${ }^{\circledR}$ II Pulsed-Field Electrophoresis System (Bio-Rad Laboratories, Nazareth, Belgium) with the following parameters: duration, $20 \mathrm{~h}$; temperature, $14^{\circ} \mathrm{C}$; first shot duration, $3.5 \mathrm{~s}$; end shot duration, $23.5 \mathrm{~s}$; shot angle, $120^{\circ}$; and current, $6 \mathrm{~V} / \mathrm{cm}^{2}$. The Pearson correlation coefficient and the unweighted pair group method with arithmetic mean were used for band and cluster analysis, respectively. Based on the Pearson correlation coefficient, strains with $\geq 95 \%$ similarity were accepted as the same clone and those with $<95 \%$ similarity were considered as different clones.

\section{Statistical Analysis}

Data were analyzed using Minitab Statistical Software version 17 (Minitab, Inc., State College, PA, USA) Descriptive statistics were presented as number (n), percentage (\%), mean and standard deviation, and median value. Kolmogorov-Smirnov test was used to determine whether the variables show normal distribution. For the comparison of continuous variables, two-sample $t$-test was used for variables showing normal distribution and MannWhitney $U$-test was used for variables not showing normal distribution. The Fisher's Exact test was used for comparison of categorical variables. A probability $(P)$ value of $\leq 0.05$ was considered statistically significant.

\section{Ethical Approval}

The study protocol was approved by the Noninterventional Clinical Research Ethics Board of Karabuk University (Date: October 25, 2016; decision no: 3/13).

\section{Results}

The demographic and clinical features of the patients with VRE are presented in Table 1. Of the 47 patients, approximately $90 \%$ were aged $\geq 60$ years (mean age, $74.6 \pm 10.6$ years; range, $43-92$ years); 26 (55.3\%) were female, and 21 (44.7\%) were male.

The mean age of the men and women was similar and was $75.7 \pm 10.6$ and $73.1 \pm 10.7$, respectively $(P=0.412)$.

Of these, $38(80.9 \%)$ received treatment in the intensive care units (ICU), whereas 9 (19.1\%) were treated in the hospital wards.

Table I Demographic and Clinical Features of Patients with $\operatorname{VRE}^{\mathrm{a}}(\mathrm{n}=47)$

\begin{tabular}{|c|c|}
\hline Data & Number (\%) \\
\hline \multicolumn{2}{|l|}{ Age (years) } \\
\hline $40-59$ & $5(10.6)$ \\
\hline $60-79$ & $25(53.2)$ \\
\hline$\geq 80$ & $17(36.2)$ \\
\hline \multicolumn{2}{|l|}{ Gender } \\
\hline Female & $26(55.3)$ \\
\hline Male & $21(44.7)$ \\
\hline \multicolumn{2}{|l|}{ Hospital unit } \\
\hline Intensive care unit (ICU) & $38(80.9)$ \\
\hline Reanimation ICU & $20(42.6)$ \\
\hline Surgical ICU & $14(29.8)$ \\
\hline Respiratory ICU & $4(8.5)$ \\
\hline Wards & $9(19.1)$ \\
\hline General surgery & $5(10.6)$ \\
\hline Urology & $4(8.5)$ \\
\hline \multicolumn{2}{|l|}{ Underlying diseases } \\
\hline Cerebrovascular disease & $23(48.9)$ \\
\hline $\mathrm{COPD}^{\mathrm{b}}$ & $20(42.6)$ \\
\hline Renal failure & $15(31.9)$ \\
\hline Hypertension & $8(17.0)$ \\
\hline Malignancy & $8(17.0)$ \\
\hline Heart failure & $6(12.8)$ \\
\hline Diabetes mellitus & $4(8.5)$ \\
\hline \multicolumn{2}{|l|}{ Comorbidities/risk factors } \\
\hline Intubation & $28(59.6)$ \\
\hline Central venous catheterization & $26(55.3)$ \\
\hline Peripheral arterial catheterization & $19(40.4)$ \\
\hline Hemodialysis & $13(27.7)$ \\
\hline Unconsciousness & $12(25.5)$ \\
\hline Total parenteral nutrition & $12(25.5)$ \\
\hline Nasogastric intubation & $10(21.3)$ \\
\hline CPR (cardiopulmonary resuscitation) & $8(17.0)$ \\
\hline Blood transfusion & $8(17.0)$ \\
\hline Steroid usage & $5(10.6)$ \\
\hline
\end{tabular}

Notes: ${ }^{a}$ Vancomycin-resistant Enterococcus. ${ }^{\mathrm{b}}$ Chronic obstructive pulmonary disease. 
Twenty-two (84.6\%) of the 26 women and $16(76.2 \%)$ of the 21 men were treated in intensive care units $(P=0.721)$

The median age of patients in the ICU was significantly higher than those in the wards and was 77 and 68, respectively $(P=0.008)$. The most common underlying diseases were cerebrovascular disease, chronic obstructive pulmonary disease (COPD), and renal failure. The most common risk factors for VRE infection were mechanical ventilation, followed by central venous and peripheral arterial catheterization.

\section{Bacterial Strains}

Twenty $(42.6 \%)$ of the 47 strains were isolated in reanimation ICU, 14 (29.8\%) from surgical ICU, and 13 (27.6\%) from 3 units ( 5 from general surgery ward, 4 from urology ward, and 4 from respiratory ICU). The 47 VRE strains were obtained from rectal $(n=38)$, urine $(n=5)$, and blood $(\mathrm{n}=4)$ samples.

Of the 38 RS, $32(84.2 \%)$ were obtained from ICU units and 6 from the wards, whereas of the 9 clinical samples $6(66.6 \%)$ were obtained from ICUs and 3 from the wards $(P=0.464)$. All 47 strains were identified as Enterococcus faecium, and all were resistant to ampicillin ( $\geq 16 \mu \mathrm{g} / \mathrm{mL})$, high-level gentamicin $(>500 \mu \mathrm{g} / \mathrm{mL})$, vancomycin $(\geq 256 \mu \mathrm{g} / \mathrm{mL})$, and teicoplanin $(\geq 32 \mu \mathrm{g} / \mathrm{mL})$. One E. faecium strain exhibited intermediate resistance to linezolid (minimum inhibitory concentration $=4 \mu \mathrm{g} / \mathrm{mL}$ ). None of the strains were resistant to daptomycin or quinupristin-dalfopristin (Q/D). All strains harbored the vanA gene but not the vanB gene.

\section{PFGE results}

The demographic data of the strains and a dendrogram of the PFGE results are presented in Figure 1. The $47 \mathrm{VRE}$ strains had 23 different profiles. Of these 47 strains, 31 were clonally related and formed 7 clusters (tolerance, 1.5; cut-off, 95\%), which are identified by Roman numerals (I-VII). Overall, 31 of the 47 strains were present in any cluster, and the clustering rate was $66 \%$. Clone I was the largest cluster with eight strains, and genotype IV was the smallest with two strains. The eight strains in clone I were isolated over a period of 6 months. Seven of the strains were obtained from ICUs and one from the urology ward. Seven strains were obtained from RS, and one from urine. Two $R S$ strains were obtained from the reanimation ICU on the same day (May 26, 2014)

Clone III consisted of seven strains and was isolated over a period of 6 months. Five of the strains were obtained from ICUs and two from patients in the urology ward. Three strains were obtained from RS, two from urine, and two from blood. Two of the three RS strains were isolated from two different ICUs on the same day (June 6). Two strains were obtained from the urine of patients in the urology ward in November 2014.

Clone VI consisted of five strains. Four of the five strains were isolated in May 2014 and one in September 2014. Four strains were obtained from RS and one from urine. Three strains were isolated from ICUs and two from wards.

Clone VII consisted of three strains. They were isolated from RS samples of patients in surgical ICU over a period of 3 days (May 2014).

\section{Discussion}

Since first reported in the United Kingdom and France in 1988, VRE has become a global health threat, particularly over the last two decades. ${ }^{6,7}$ E. faecalis is the most frequently isolated species from clinical specimens, and a high level of antibiotic resistance is common in E. faecium. ${ }^{4,6,7}$ Because of the limited treatment options, in 2017, the World Health Organization recently classified vancomycin-resistant Enterococcus faecium (VREfm) as a high-priority pathogen group requiring urgent development of new antibiotics. ${ }^{12}$

In this study, all 47 VRE strains were identified as E. faecium and harbored the vanA resistance gene but not the vanB gene. Moreover, consistent with the vanA genotype, all strains were highly resistant to vancomycin and teicoplanin. Similarly, Gözalan et al reported that all 55 VREfm strains carried the vanA gene. ${ }^{13}$ In other studies, from Turkey, the rate of vanA carriage has been determined to be $84-100 \%$. $^{14-16}$

The vanA type resistance is dominant in the United States and Europe, whereas vanB type resistance is more frequent in Australia and Southeast Asia., ${ }^{77}$ However, VRE strains carrying vanB or both vanA/vanB have been reported in various countries. ${ }^{17-19}$ In Turkey, the first Van B carrying VRE strain was reported in $2012 .{ }^{20}$ The carriage of vanB ranged from $2 \%$ to $13.5 \%$ in Turkey. ${ }^{14,15,20}$ Also, both vanA and vanB gene carrying VRE strains have been reported. ${ }^{14,15}$ In the present study, all the strains were resistant to ampicillin and high-level gentamicin. Enterococci are intrinsically resistant to low levels of aminoglycoside antibiotics (AGA). ${ }^{1,4,5}$ Therefore, AGAs combined with beta-lactam antibiotics inhibit the synthesis of the cell wall and ribosomal proteins by passing through the damaged bacterial cell wall, which results in 


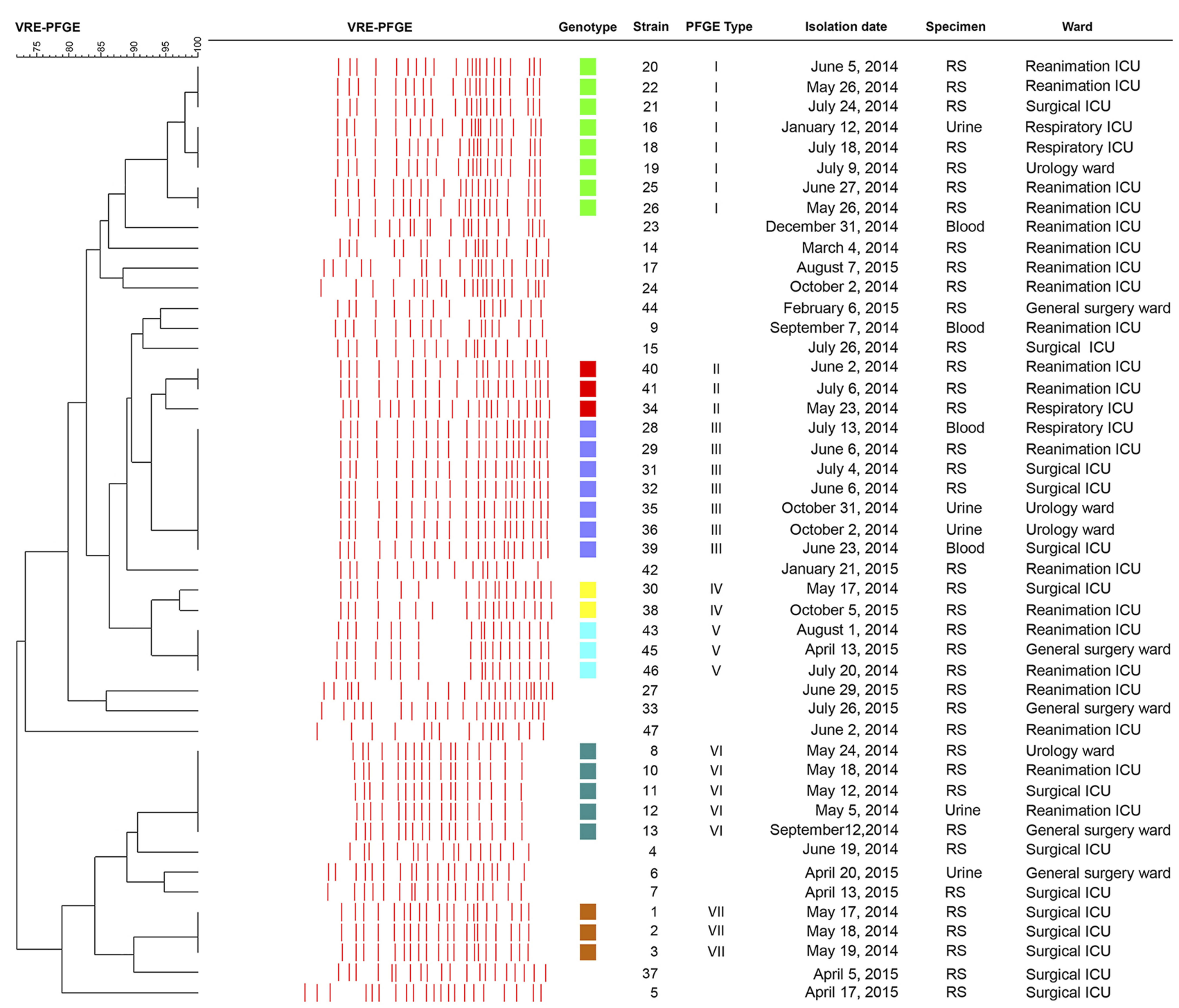

Figure I Dendrogram based on pulsed-field gel electrophoresis pattern analysis of the VRE strains. The distribution of PFGE types among VRE strains and other demographic data (specimen, isolation date, and wards) are presented. The PFGE genotypes of VRE strains were named with Roman numerals (I-VII).

Abbreviations: RS, rectal swab; ICU, intensive care unit.

a synergistic effect. ${ }^{2,4}$ However, in the presence of highlevel AGA resistance (MIC $>2000 \mu \mathrm{g} / \mathrm{mL}$ for streptomycin and $500 \mu \mathrm{g} / \mathrm{mL}$ for gentamicin) this synergy abolishes. High-level AGA resistance in enterococci has been reported to be of transferable type, mediated by AGAmodifying enzymes. ${ }^{4,6}$

The rate of high-level gentamicin resistance (HLGR) in E. faecium is reportedly low in Cyprus (4.9\%) and Luxemburg (12.9\%) but relatively high in Turkey (52.3\%), Iran $(74.9 \%)$, and Korea $(91.6 \%){ }^{21-24}$ According to Central Asia and Eastern European Surveillance of Antimicrobial Resistance data, $65 \%-95 \%$ of E. faecium isolates are resistant to ampicillin and $81 \%-97 \%$ are classified as $\mathrm{HLGR}^{25}$ In India, the rates of ampicillin resistance and HLGR are $80 \%$ and $77.1 \%$, respectively. ${ }^{26}$ Similarly, a study conducted in Australia by Coombs et al. ${ }^{17}$ reported ampicillin resistance and HLGR rates of $90.4 \%$ and $65 \%$, respectively, indicating that the combination of ampicillin and AGA is no longer an effective treatment option for VREfm infections in these countries.

In the current study, only one isolate was intermediately resistant to linezolid. Because of increased VRE infections, linezolid was used more frequently to treat the infections. Linezolid is an oxazolidinone derivative that prevents the formation of the bacterial $70 \mathrm{~S}$ ribosome by binding is to peptidyl transferase, which inhibits protein synthesis. ${ }^{1,7}$ Moreover, it has a bacteriostatic effect against both E. faecalis and E. faecium species. ${ }^{27}$ In 2000 , the US 
Food and Drug Administration approved linezolid for the treatment of skin and soft tissue infections, nosocomial pneumonia, and VREfm infections. 6 ,7

Linezolid resistance in Enterococcus species is related to a mutation in the $23 \mathrm{~S}$ rRNA gene region as well as the presence of the plasmid-encoded chloramphenicol-florfenicol resistance (cfr) gene. ${ }^{6,27,28}$

According to various studies, the rate of linezolid resistance in enterococci is low in Iran (0.7\%), Turkey $(1 \%)$, and Australia $(1.3 \%)^{17,23,29}$ but relatively high in Canada $(13.7 \%)^{30}$

In this study, all VREfm strains were susceptible to daptomycin. Daptomycin is a lipopeptide derivative that irreversibly binds to the bacterial cytoplasmic membrane and disrupts intracellular ion balance. ${ }^{4,28}$ Since 2006, daptomycin has been used in Europe for the treatment of Staphylococcus aureus-related skin and soft tissue infections $^{1,31}$ and as an alternative treatment for VRE infection. However, the development of daptomycin resistance during treatment has been reported. ${ }^{7,31}$ Daptomycin resistance of enterococci has been found to be related to the mutations that are caused by amino acid changes in the LiaFSR signaling system, which controls the cell membrane stress response. ${ }^{1,6,28}$ In a surveillance study conducted in the US, $0.2 \%$ and $3.9 \%$ of E. faecalis and E. faecium isolates were resistant to daptomycin, respectively. ${ }^{7}$

Daptomycin resistance rates of enterococci varied from $7 \%$ in Ireland to $8.2 \%$ in France and $10.5 \%$ in Spain, whereas no daptomycin resistance has been reported to date in Norway, Poland, and Portugal. ${ }^{4,28}$

The $\mathrm{Q} / \mathrm{D}$ is active against $E$. faecium but not E. faecalis. ${ }^{1,4}$ In this study, all VREfm strains were susceptible to Q/D [70\% dalfopristin (streptogramin A) and 30\% quinupristin (streptogramin B)]. Similarly, Gözalan et al reported that all 55 VREfm strains were Q/D sensitive. ${ }^{13}$ However, the rate of $\mathrm{Q} / \mathrm{D}$ resistance among VREfm strains has been reported to be $3 \%-7.2 \%$ in Turkey. ${ }^{20,32,33}$ In a multi-center surveillance study, the rates of $Q / D$ resistance of VREfm strains in North America and the European Union were $0.6 \%$ and $10 \%$, respectively. ${ }^{34}$ Furthermore, reduced susceptibility to $\mathrm{Q} / \mathrm{D}$ in other countries reportedly ranges from $17.6 \%$ to $28.6 \%{ }^{35}$

The most common $\mathrm{Q} / \mathrm{D}$ resistance of enterococci occurs with macrolide-lincosamide-streptogramin B (MLSb), which is mediated by the erm genes. ${ }^{8}$ Owing to changes in the $23 \mathrm{~S}$ ribosomal region, the dimethylase enzyme encoded by these genes reduces the binding of the MLSb-group antibiotics to the target region. ${ }^{4,8}$ Streptogramin A resistance is exhibited in the form of enzymatic inactivation of the antibiotic, which is mediated by the vatD and vatE genes. Furthermore, Streptogramin B resistance is associated with the active efflux system, which is mediated by the $m s r C$ gene. $^{1,4,35}$

In the literature, various risk factors related to VRE colonization include exposure to invasive procedures (urinary catheterization, intravenous or central venous catheterization, and mechanical ventilation), prolonged length of stay in the ICU, immunosuppression, chronic renal failure, and history of antibiotic use (particularly vancomycin, third-generation cephalosporins, and metronidazole). ${ }^{1,3,6,36}$ Similarly, all patients included in this study had more than one underlying disease/risk factor. The most frequent underlying diseases were cerebrovascular disease $(55 \%)$, COPD (43\%), and renal failure $(32 \%)$, whereas $60 \%$ had received mechanical ventilation support, 55\% had a central venous catheter, and $40 \%$ had a peripheral arterial catheter.

When an outbreak is suspected or there is an increase in infection rates, it is necessary to investigate the clonal relationship among strains to detect the source of infection and route of transmission. In the current study, the clonal relatedness among strains was evaluated using PFGE. The PCR-based molecular typing methods such as arbitrarily primed-PCR and repetitive extragenic palindromic-PCR have been used to determine the epidemiological characteristics of VRE outbreaks. Although there have some limitations, PFGE is still considered to be the gold standard method, because the results can be quantified with high discriminatory power and repeatability. ${ }^{13,37-40}$ According to the PFGE results, no common clone outbreak occurred in our hospital, but polyclonal spread was observed, and high clustering rate indicated cross-contamination. Multiclonal spread leading to small outbreaks was observed from time to time. For instance, three strains of clone VII were obtained from RSs taken at the same time (May 2014) from three patients in the surgical ICU. Two strains of clone III were obtained from urine cultures of patients in the urology ward in October 2015. As the majority of the clonally related strains were obtained from the reanimation and surgical ICUs, it was understood that these units were the main reservoirs. However, smaller clonal spreads were observed within departments. In accordance with our results, polyclonal spread of VRE strains was reported in Tunisia $^{3}$ and Australia. ${ }^{17}$ In addition, several other studies have reported single clone outbreaks in Turkey $^{11,13}$ and Italy. ${ }^{37}$ 
The retrospective, monocentric study design and small sample size constitute our limitations. Besides, virulence determinants of VRE isolates were not investigated.

\section{Conclusion}

The incidences of VRE-related infections continue to increase worldwide. Moreover, the emergence of resistance to linezolid and daptomycin are a matter of great concern. To prevent VRE infections, effective and comprehensive precautions must be taken. These include antibiotic stewardship to reduce VRE colonization in high-risk patients, infection control measures to prevent cross-transmission, active microbiologist-clinician collaboration, and the implementation of rapid and accurate VRE detection methods in the laboratory. ${ }^{1,2,4}$

The results of this study revealed that van A harboring E. faecium was predominant in our hospital and that polyclonal VRE spread had occurred within the hospital units. The high clustering rate among the VRE strains indicates crosstransmission. Therefore, effective and multifaceted infection control policies should be implemented in our hospital.

\section{Acknowledgments}

This study was funded by the Karabuk University Scientific Research Project Unit (project number: KBU-BAP-KT-061). This study was presented at the International DNA Day And Genome Congress (April 24-28, 2017, Ahi Evran University, Kirsehir, Turkey). The authors would like to thank Professor Seyit Ali Kayis for statistical analysis.

\section{Disclosure}

The authors report no conflicts of interest in this work.

\section{References}

1. Cesar AA, Murray BE. Enterococcus species, streptococcus gallolyticus group, and leuconostoc species. In: Bennet JE, Dolin R, Blaser MJ, editors. Mandell, Douglas, and Bennett's Principles and Practice of Infectious Diseases. Philadelphia (PA): Elsevier Inc.; 2015:2328-2339.

2. Reyes K, Bardossy AC, Zervos M. Vancomycin-resistant enterococci: epidemiology, infection prevention, and control. Infect Dis Clin North Am. 2016;30(4):953-965. doi:10.1016/j.idc.2016.07.009

3. Dziri R, Lozano C, Ben Said L, et al. Multidrug-resistant enterococci in the hospital environment: detection of novel vancomycin-resistan E. faecium clone ST910. J Infect Dev Ctries. 2016;10(8):799-806. doi: $10.3855 /$ jidc. 8014

4. Faron ML, Ledeboer NA, Buchan BW, Kraft CS. Resistance mechanisms, epidemiology, and approaches to screening for vancomycin-resistant enterococcus in the health care setting. J Clin Microbiol. 2016;54 (10):2436-2447. doi:10.1128/JCM.00211-16

5. Hollenbeck BL, Rice LB. Intrinsic and acquired resistance mechanisms in enterococcus. Virulence. 2012;3(5):421-433. doi:10.4161/viru.21282
6. O'Driscoll T, Crank CW. Vancomycin-resistant enterococcal infections: epidemiology, clinical manifestations, and optimal management. Infect Drug Resist. 2015;8:217-230. doi:10.2147/IDR.S54125

7. Guzman Prieto AM, van Schaik W, Rogers MRC, et al. Global emergence and dissemination of enterococci as nosocomial pathogens: attack of the clones?. Front Microbiol. 2016;7(5):1-15. doi:10.3389/fmicb.2016.00788

8. Yang JX, Li T, Ning YZ, et al. Molecular characterization of resistance, virulence and clonality in vancomycin-resistant enterococcus faecium and enterococcus faecalis: A hospital-based study in Beijing, China. Infect Genet Evol. 2015;33:253-260. doi:10.1016/j. meegid.2015.05.012

9. CLSI. Performance Standards for Antimicrobial Susceptibility Testing. $27^{\text {th }}$ Informational Supplement. Wayne, PA: Clinical and Laboratory Standards Institute; 2017

10. Nasaj M, Mousavi SM, Hosseini SM, Arabestani MR. Prevalence of virulence factors and vancomycin-resistant genes among Enterococcus faecalis and E. faecium isolated from clinical specimens. Iran J Public Health. 2016;45(6):806-813.

11. Güldemir D, Karagoz A, Dal T, Tekin A, Ozekinci T, Durmaz R. Molecular typing of nosocomial enterococci by pulsed-field gel electrophoresis. Turk Hij Den Biyol Derg. 2015;72(1):1-10. doi:10. 5505/TurkHijyen.2015.94695

12. World Health Organization (WHO). WHO Publishes List of Bacteria for Which New Antibiotics are Urgently Needed. Geneva, Switzerland; 2017. Available from https://www.who.int/news-room/detail/27-02-2017-whopublishes-list-of-bacteria-for-which-new-antibiotics-are-urgently-needed. Accessed April 18, 2019.

13. Gozalan A, Coskun-Ari FF, Ozdem B, et al. Molecular characterization of vancomycin-resistant Enterococcus faecium strains isolated from carriage and clinical samples in a tertiary hospital, Turkey. J Med Microbiol. 2015;64(7):759-766. doi:10.1099/jmm.0.000088

14. Terkuran M, Erginkaya Z, Unal E, Guran M, Kizilyildirim S, Gökce U. The relationship between virulence factors and vancomycin resistance among Enterococci collected from food and human samples in Southern Turkey. Ankara Univ Vet Fak Derg. 2014;61:133-140.

15. Uludag HA, Ataman $\mathrm{CH}$, Bulut C, Eser OK, Demiröz AP. Comparison of GeneXpert ${ }^{\circledR}$ vanA/vanB PCR system and culture methods in the surveillance of vancomycin-resistant enterococci. Mikrobiyol Bul. 2014;48(4):538-544. doi:10.5578/mb.8848

16. Arslan U, Demir E, Oryasin E, et al. MLST types of vancomycin-resistant enterococcus faecium strains isolated from blood cultures. Mikrobiyol Bul. 2013;47(3):432-441. doi:10.5578/ mb.5085

17. Coombs GW, Pearson JC, Daley DA, et al. Molecular epidemiology of enterococcal bacteremia in Australia. $J$ Clin Microbiol. 2014;52:897-905. doi:10.1128/JCM.03286-13

18. Marcadé G, Micol J, Jacquier H, et al. Outbreak in a haematology unit involving an unusual strain of glycopeptide-resistant Enterococcus faecium carrying both vanA and vanB genes. J Antimicrob Chemother. 2014;69(2):500-505. doi:10.1093/jac/dkt376

19. Papagiannitsis CC, Malli E, Florou Z, et al. First description in Europe of emergence of enterococcus faecium ST117, carrying both vanA/vanB genes, isolated in Greece. J Glob Antimicrob Resist. 2017;11:68-70. doi:10.1016/j.jgar.2017.07.010

20. Coskun FA, Mumcuoglu I, Aksu N, et al. Phenotypic and genotypic traits of vancomycin-resistant enterococci in a public hospital: the first vanB-positive Enterococcus faecium isolates. Mikrobiyol Bul. 2012;46(2):276-282.

21. European Centre for Disease Prevention and Control. Surveillance Atlas of Infectious Diseases [Internet]. Stockholm: ECDC; 2018. Available from: http://atlas.ecdc.europa.eu. Accessed June 12, 2019

22. Kilbas I, Ciftci IH. Antimicrobial resistance of Enterococcus isolates in Turkey: a meta-analysis of current studies. J Glob Antimicrob Resist. 2018;12:26-30. doi:10.1016/j.jgar.2017.08.012 
23. Asadollahi P, Razavi S, Asadollahi K, et al. Rise of antibiotic resistance in clinical enterococcal isolates during 2001-2016 in Iran: a review. New Microbes New Infect. 2018;26:92-99. doi:10.1016/j.nmni.2018.08.018

24. Oh WS, Ko KS, Song $\mathrm{JH}$, et al. High rate of resistance to quinupristin-dalfopristin in Enterococcus faecium clinical isolates from Korea. Antimicrob Agents Chemother. 2005;49(12):5176-5178. doi:10.1128/AAC.49.12.5176-5178.2005

25. Central Asian and Eastern European Surveillance of Antimicrobial Resistance (CAESAR) (2018). Annual Report 2017. Available from: http://www.euro.who.int/en/health-topics/disease-prevention/antimicro bial-resistance/publications/2017/central-asian-and-eastern-european-sur veillance-of-antimicrobial-resistance--annual-report-2017-2018. Accessed March 28, 2019.

26. Nayak PP, Biranthabail D, Shenoy S, Kotian SM. Antibiogram and genetic relatedness of clinical isolates of Enterococcus spp. in Mangalore, India. J Infect Dev Ctries. 2018;12(11):985-990. doi:10.3855/ jidc. 9966

27. Bi R, Qin T, Fan W, Ma P, Gu B. The emerging problem of linezolidresistant enterococci. J Glob Antimicrob Resist. 2018;13:11-19. doi:10.1016/j.jgar.2017.10.018

28. Bender JK, Cattoir V, Hegstad K, et al. Update on prevalence and mechanisms of resistance to linezolid, tigecycline and daptomycin in enterococci in Europe: towards a common nomenclature. Drug Resist Updat. 2018;40:25-39. doi:10.1016/j.drup.2018.10.002

29. Moosavian M, Ghadri H, Samli Z. Molecular detection of van A and van B genes among vancomycin-resistant enterococci in ICU hospitalized patients in Ahvaz in southwest of Iran. Infect Drug Resist. 2018;11:2269-2275. doi:10.2147/IDR.S177886

30. Simner PJ, Adam H, Baxter M, et al. Epidemiology of vancomycinresistant Enterococci in Canadian hospitals (CANWARD Study, 2007 to 2013). Antimicrob Agents Chemother. 2015;59(7):4315-4317. doi:10. 1128/AAC.00384-15

31. Lellek H, Franke GC, Ruckert C, et al. Emergence of daptomycin non-susceptibility in colonizing vancomycin-resistant Enterococcus faecium isolates during daptomycin therapy. Int $\mathrm{J}$ Med Microbiol. 2015;305(8):902-909. doi:10.1016/j.ijmm.2015.09.005
32. Baylan O, Nazik H, Bektöre B, et al. The relationship between antibiotic resistance and virulence factors in urinary Enterococcus isolates. Mikrobiyol Bul. 2011;45(3):430-445.

33. Baysallar M, Kilic A, Aydogan H, Cilli F, Doganci L. Linezolid and quinupristin/dalfopristin resistance in vancomycin-resistant enterococci and methicillin-resistant Staphylococcus aureus prior to clinical use in Turkey. Int J Antimicrob Agents. 2004;23(5):510-512. doi:10.1016/j. ijantimicag.2003.09.029

34. Mercuro NJ, Davis SL, Zervos MJ, Herc ES. Combatting resistant enterococcal infections: a pharmacotherapy review. Expert Opin Pharmacother. 2018;19(9):979-992. doi:10.1080/14656566.2018. 1479397

35. Isogai N, Urushibara N, Kawaguchiya M, et al. Characterization of enterococcus faecium with macrolide resistance and reduced susceptibility to quinupristin/dalfopristin in a Japanese hospital: detection of extensive diversity in erm(B)-regulator regions. Microb Drug Resist. 2013;19(4):298-307. doi:10.1089/mdr.2012.0176

36. Monteserin N, Larson E. Temporal trends and risk factors for healthcare-associated vancomycin-resistant enterococci in adults. J Hosp Infect. 2016;94(3):236-241. doi:10.1016/j.jhin.2016.07.023

37. Bressan R, Knezevich A, Monticelli J, et al. Spread of vancomycin-resistant Enterococcus faecium Isolates despite validated infection control measures in an Italian hospital: antibiotic resistance and genotypic characterization of the endemic strain. Microb Drug Resist. 2018;24(8):1148-1155. doi:10.1089/mdr.2017.0314

38. Lytsy B, Engstrand L, Gustafsson Å, Kaden R. Time to review the gold standard for genotyping vancomycin-resistant enterococci in epidemiology: comparing whole-genome sequencing with PFGE and MLST in three suspected outbreaks in Sweden during 2013-2015. Infect Genet Evol. 2017;54:74-80. doi:10.1016/j.meegid.2017.06.010

39. Pulsed-field Gel Electrophoresis (PFGE). Centers for Disease Control and Prevention. Available from: https://www.cdc.gov/pulsenet/patho gens/pfge.html. Accessed October 14, 2019.

40. Benammar S, Pantel A, Aujoulat F, et al. First molecular characterization of related cases of healthcare-associated infections involving multidrug-resistant Enterococcus faecium vanA in Algeria. Infect Drug Resist. 2018;11:1483-1490. doi:10.2147/IDR.S164487
Infection and Drug Resistance

\section{Publish your work in this journal}

Infection and Drug Resistance is an international, peer-reviewed openaccess journal that focuses on the optimal treatment of infection (bacterial, fungal and viral) and the development and institution of preventive strategies to minimize the development and spread of resistance. The journal is specifically concerned with the epidemiology of antibiotic resistance and the mechanisms of resistance development and diffusion in both hospitals and the community. The manuscript management system is completely online and includes a very quick and fair peerreview system, which is all easy to use. Visit http://www.dovepress.com/ testimonials.php to read real quotes from published authors. 